\title{
Pharmaconutrition administration on outcomes of elective oncological surgery for gastrointestinal malignancies: is timing everything? - a review of published meta-analyses until the end of 2016
}

\author{
Emma Osland $^{1,2}$, Breda Memon ${ }^{3}$, Muhammed Ashraf Memon $^{3,4,5,6,7}$ \\ ${ }^{1}$ Department of Nutrition, Royal Brisbane and Women's Hospital, Brisbane, Queensland, Australia; ${ }^{2}$ School of Human Movement and Nutrition \\ Sciences, University of Queensland, Brisbane, Queensland, Australia; ${ }^{3}$ Sunnybank Obesity Centre, McCullough Centre, Sunnybank, Queensland, \\ Australia; ${ }^{4}$ Mayne Medical School, School of Medicine, University of Queensland, Brisbane, Queensland, Australia; ${ }^{5}$ Faculty of Health Sciences and \\ Medicine, Bond University, Gold Coast, Queensland, Australia; ${ }^{6}$ School of Agricultural, Computational and Environmental Sciences, International \\ Centre for Applied Climate Sciences and Centre for Health Sciences Research, University of Southern Queensland, Toowoomba, Queensland, \\ Australia; ${ }^{7}$ Faculty of Health and Social Science, Bolton University, Bolton, Lancashire, UK \\ Contributions: (I) Conception and design: All authors; (II) Administrative support: All authors; (III) Provision of study materials or patients: All \\ authors; (IV) Collection and assembly of data: All authors; (V) Data analysis and interpretation: All authors; (VI) Manuscript writing: All authors; (VII) \\ Final approval of manuscript: All authors. \\ Correspondence to: Prof. Muhammed Ashraf Memon. South East Queensland Surgery (SEQS) and Sunnybank Obesity Centre, Suite 9, McCullough \\ Centre, 259 McCullough Street, Sunnybank, QLD 4109, Australia. Email: mmemon@yahoo.com.
}

\begin{abstract}
The last 25 years have seen an increasing number of publications attesting the benefits of pharmaconutrition in the management of patients undergoing elective oncological gastrointestinal surgery. A number of randomized controlled trials and meta-analyses suggest the use of pharmaconutrition in this group of patients produces superior outcomes to standard nutritional formulations in terms of postoperative infective complications, anastomotic breakdown and length of hospital stay. The use of pharmaconutrition products, therefore, has gained increasing acceptance for use in elective gastrointestinal oncological surgical populations and been incorporated into practice guidelines. However, there remains doubts as to the robustness of such data supporting these recommendation. This is because studies reporting improved outcomes with pharmaconutrition (I) frequently compare this intervention with non-equivalent control groups; (II) do not report on the actual nutritional provision received by study participants; (III) overlook the potential impact of industry funding on research conducted and (IV) do not adopt a multidisciplinary approach to the research undertaken. For these reasons, a critical re-appraisal of the use and recommendations of pharmaconutrition in this group of patients is urgently warranted to resolve some of the above mentioned issues. The aim of this review was to analyse meta-analyses published until the end of 2016 in this area to highlight the strengths and weakness of the present research and prioritize certain areas which will benefit from future research.
\end{abstract}

Keywords: Pharmaconutrition; immunonutrition (IN); gastrointestinal surgery; gastrointestinal malignancies; elective surgery; cancer; complications; meta-analysis; systematic review; human

Received: 19 March 2018; Accepted: 30 July 2018; Published: 08 August 2018.

doi: $10.21037 / \operatorname{tgh} .2018 .07 .12$

View this article at: http://dx.doi.org/10.21037/tgh.2018.07.12 


\section{Introduction}

Nutrition provision is recognized to be an important aspect in the perioperative management of elective oncologic surgery for gastrointestinal malignancy patients, because the timely provision of nutrition has been shown to be associated with improved postoperative outcomes $(1,2)$. These benefits are thought to arise from the provision of macronutrients such as calories for energy and protein for wound healing, and this in turn reduces the impact of catabolism in the postoperative period. However, more recently it has been theorized that due to the complex postoperative inflammatory, immune and oxidative stress, providing specific nutrients in supraphysiological doses may provide vital substrates that serve to modulate these immune and metabolic responses and thus improve clinical outcomes (3). In view of this, during the early 1990s new nutrition support formulas emerged containing higher quantities of arginine, with or without glutamine, omega-3 fatty acids, and nucleotides (3). These products have been commonly referred to as 'immunonutrition', 'immune-enhancing diets', and more recently as 'pharmaconutrition' in recognition of their intended pharmaceutical-like action rather than purely as nutrient provision (3).

In an elective surgical population with gastrointestinal malignancies, the use of pharmaconutrition has been reported to reduce postoperative infective complications and hospital length of stay (LOS), without adversely affecting mortality described in medical and trauma subgroups of a critically ill population (4-10). The results of individual studies have been conflicting (11-15), however the use of these products gain increasing acceptance following their incorporation into practice guidelines $(16,17)$. As of 2016 eleven meta-analyses on this topic have been conducted on purely surgical patients, the majority of which are receiving surgery for oncological conditions (18-25) or with surgical patients as a subgroup of critical ill population (26-28). However there are a number of limitations to applying the outcomes of some of these meta-analyses to practice due to the inclusion of studies utilizing non-equivalent control groups, inclusion of diverse surgical populations, and the failure to account for practical differences between the studies (i.e., administration protocols of pharmaconutrition). The present review will provide an overview and critique of the "meta-analyses" published in the English literature to the end of 2016 on the topic of pharmaconutrition on elective oncological surgical patients.

\section{Appraisal of meta-analyses}

The first three meta-analyses on the subject of pharmaconutrition were published between 1999 and 2001. Heys et al. (26), Heyland et al. (27) and Beale et al. (28) all include elective surgical patients as a subgroup analysis of meta-analyses on the critically ill patients suffering from various conditions such as sepsis, burns, and trauma. All these three meta-analyses demonstrated that pharmaconutrition leads to lower infectious complication rates and hospital stay, however, it does not impact an overall mortality. Nonetheless, the question remains, are these results really applicable to purely surgical patients especially those undergoing elective gastrointestinal oncologic surgery?

Since the publication of these three meta-analyses (26-28), many more RCTs have been published on the role of pharmaconutrition $v s$. standard enteral nutrition (SEN) exclusively on elective surgical populations. Waitzberg et al. (18) conducted a meta-analysis on RCTs published before 2003 that utilized the commercially available product, Impact ${ }^{\circledR}$ (Novartis Consumer Health, Switzerland). The authors included 17 RCTs (2,305 patients) published between 1988 and 2000 in various languages analysing pre- and postoperative enteral nutrition with and without Impact ${ }^{\circledR}$. The authors of this study concluded that Impact ${ }^{\circledR}$ at a certain dosage (0.5-1 L/day) and duration (5-7 days preoperatively) contributes to significantly improved outcome of morbidity in elective oncological surgical patients along with decreased length of hospital stay. Their meta-analysis suggested 39\% to $61 \%$ reductions in postoperative infectious complications and $46 \%$ decreased in anastomotic leak rate. Furthermore, they concluded that the cost effectiveness of such practice is supported by health economic analysis. However, there are a number of biases introduced in this meta-analysis which includes (I) inclusion of cardiac surgery patients with an otherwise largely gastrointestinal surgery cohort and (II) inclusion of studies that utilized non-equivalent control groups such as intravenous fluids or crystalloids, or nil-by-mouth. The heterogeneity introduced through these inclusions, the exclusion of studies conducted using other similarly composed commercial products, and the suggestion that this meta-analysis has been funded by the company producing Impact ${ }^{\circledR}$ result in the need to interpret the outcomes of this analysis with caution.

Zheng et al. (21) restricts inclusion criteria to 
gastrointestinal surgery (both upper and lower) but makes no attempt to control for the differences within the administration of pharmaconutrition between studies. The aim of their meta-analysis was to evaluate clinical and economic validity of perioperative immunonutrition (IN) and effect on postoperative immunity in these patients. They analysed 13 RCTs (1,269 patients) mainly for upper GI pathologies including cancer between 1992 and 2005. They concluded that IN, like previous meta-analyses, had no significant effect on postoperative mortality, however, it significantly reduces the risk of postoperative infection, length of hospital stay and improves immune function by increasing total lymphocytes, CD4 levels and decreasing IL6 levels. They highlighted the fact, that perioperative IN is effective and safe in decreasing postoperative infection and length of hospital stay by improving immunity of postoperative patients compared to SEN. However, given the lack of consideration regarding the timing of administration of IN on the outcomes reported, the validity of their analysis and conclusions to guide practice is questionable.

Marik and Zaloga (19) published a systematic review of 21 RCTs published between 1992 and 2008 and totalling 1,918 patients. The authors were comparing the effect of arginine and/or omega-3 containing pharmaconutrition products with standard formulations. While the majority of inclusions were for gastrointestinal malignancies, they included two RCTs on head and neck malignancy patients and one on cardiac surgery patients. The authors included a priori analyses on differing compositions and timing of pharmaconutrition. The authors themselves conceded that the optimal timing cannot be determined from their analysis, however they feel that preoperative therapy may be of benefit although no rationale is provided. Their results are difficult to apply to practice, due to the heterogeneous surgical populations included (head and neck, cardiac and gastrointestinal) and the significant methodological flaw of performing meta-analysis statistics in instances where only one study met the inclusion criteria (29).

Cerantola et al. (20) published another meta-analysis on IN in gastrointestinal surgery in 2011. They included RCTs published between 1985 and 2009 evaluating the clinical impact of peri-operative enteral pharmaconutrition (including those of varying formulae) in major gastrointestinal elective surgery. Twenty-one RCTs totalling 2,730 patients were evaluated in patients receiving preor pre- and post-or postoperative pharmaconutrition $v s$. SEN. This paper incorporated RCTs on an exclusively gastrointestinal surgical population, addressed the timing of pharmaconutrition provision through performing subgroup analyses, and is the first meta-analysis on this topic to comply with PRISMA reporting guidelines. However, it also includes studies that use non-equivalent control groups $(7,8,10,30)$ which may produce outcomes that appear to favour pharmaconutrition independent of the role of immune-enhancing components. They concluded that perioperative enteral pharmaconutrition decreased morbidity and hospital stay but not mortality after major gastrointestinal surgery and therefore its routine use can be recommended.

Osland et al. (22) investigated the impact on postoperative outcomes from RCTs comparing arginine-dominant pharmaconutrition formulations with standard products in elective gastrointestinal malignancy surgical population. This meta-analysis differs from those preceding it through the emphasis placed on timing of the pharmaconutrition provided and through use of stricter inclusion criteria to reduce heterogeneity in the results obtained. Twenty RCTs published between 1980 and 2011 ( $n=2,005$ patients) were included in the analysis. It not only confirms previous findings regarding arginine-dominant pharmaconutrition but provides further insight into the effects of its use. Firstly, it continues to show no adverse effect on postoperative mortality in elective gastrointestinal surgical populations. It also supports the commonly accepted benefits of argininedominant pharmaconutrition with relation to reductions in postoperative infective complications, however these benefits were only seen in peri- and postoperative pharmaconutrition administration. Similarly, reductions in length of hospital stay were noted in peri- and postoperative administration, however heterogeneity evidenced by a high $\mathrm{I}^{2}$ index and publication bias present in this data makes it difficult to draw tangible conclusions on the positive impact over some of these variables. Distinct differences in the attributed benefits of pharmaconutrition and the timing of its administration were an important finding of this meta-analysis. Previous meta-analyses performing a priori analyses on timing of pharmaconutrition report benefit irrespective of when in the clinical course it is provided $(19,20)$. One notable exception is that preoperative pharmaconutrition was not shown to reduce length of hospital stay as suggested by Cerantola et al. (20). Osland et al. (22) meta-analysis demonstrates no benefit from the provision of preoperative pharmaconutrition across any of the outcomes assessed. A possible explanation for this is the stricter inclusion criteria applied to minimize 
heterogeneity. Thus the results reported may be a truer indication of the effect of preoperative pharmaconutrition in this surgical population. The pharmacokinetics of pharmaconutrients may assist in understanding this finding. Serum arginine levels have been shown to significantly increase following seven days of preoperative $(12,31)$ and postoperative administration $(31,32)$. Sustained elevated serum levels have been demonstrated at postoperative day 8 with perioperative administration (33). However, no study appears to have investigated the postoperative serum levels of patients receiving preoperative pharmaconutrients as a standalone intervention. It is therefore conceivable that the cessation of pharmaconutrition on the day of surgery may result in sub-therapeutic or declining levels of circulating pharmaconutrients within the postoperative period when their action may be most valuable. Beta-error (false negative) may also play a part in the findings reported in this and/or previous meta-analyses given the small number of studies investigating preoperative pharmaconutrition interventions.

Osland et al. (22) further suggests that pharmaconutrition may provide additional benefits in terms of reduction of anastomotic dehiscence and non-infective complications in perioperative and postoperative administration respectively - these phenomena have not previously been reported in association with arginine-dominant pharmaconutrition. Reduced non-infectious complications in postoperative pharmaconutrition provision may potentially be explained by the higher caloric and/ or nitrogen content of many of the pharmaconutrition formulations when compared to the control formulations. Six of the fourteen studies ( $42 \%$ ) included in the postoperative meta-analysis use intervention products that contain between $20 \%$ and $46 \%$ more protein $(11,14,34-37)$ and/or up to $600 \mathrm{kcal}(20 \%)$ more energy (14) than the control formulations. In a gastrointestinal oncological surgical population who traditionally present with a high prevalence of malnutrition, the higher overall nutritional provision may be enough to account for this unexpected finding given that malnourished patients experience more profound improvements in clinical outcomes attributable to nutritional provision than their well-nourished counterparts (38). This explanation, however, does not adequately explain the reduced anastomotic dehiscence reported with the perioperative administration of pharmaconutrition as these used comparable products for both arms of their studies. As leukocytosis is recognized as a risk factor for anastomotic dehiscence (39) it seems plausible that the reduction in infective complications associated with pharmaconutrition may provide additional protection in the surgical anastomosis through this mechanism. However, given the small number of perioperative studies analysed, beta-error may also be a plausible explanation for this finding.

The potential of variations in the composition of included pharmaconutrition products to confound results observed are a limitation of the Osland et al. (22) metaanalysis. The authors defend their decision to include a diverse range of arginine-containing products in their analysis as arginine has been the most consistently utilized pharmaconutrient in elective gastrointestinal surgical populations, and remains the consistent ingredient that links commercial and experimental formulas in this genre of products. However this meta-analysis (22) contributes to the literature on this topic by utilizing stricter inclusion criteria with regards to the control group (as far as the literature allows), and to exclusively analyse studies according to the timing of pharmaconutrition delivery. We believe this issue is of vital importance to guide the translation of research to clinical practice.

Hegazi et al. (23) undertook a systematic review of 8 RCTs of preoperative IN versus standard oral nutritional supplements (ONS) and 9 RCTs of IN $v$ s. no supplements (NS). The study population consisted of patients undergoing upper or lower gastrointestinal surgery predominantly for the curative management of cancer. There was a mixed patient population in various RCTs comprising of malnourished to well-nourished patients. According to the authors, compared to standard ONS, preoperative IN was not associated with reduced wound infection, all infectious complications, non-infectious complications or length of hospital stay. However, in RCTs comparing preoperative IN vs. NS, preoperative IN was associated with significant decreased infectious complications and length of hospital stay. The authors of this meta-analysis highlighted two key areas of concern (I) lack of a clear definition of malnutrition and (II) inclusion of well-nourished patients in some of these RCTs. They also highlighted the varied composition and individual nutrients of the standard ONS especially the amount and biologic value of protein contained which is critical to help promote muscle protein synthesis and decreased inflammation associated loss of lean body mass and function. The authors concluded that ONS and IN are comparable in their effects on several key clinical outcomes, however the authors resisted in concluding that ONS results in improved outcomes. They also stressed 
the fact that standard ONS are less expensive and widely available which improves patient compliance.

Song et al. (24) published the largest meta-analysis to 2016 in the area of pharmaconutrition for patient undergoing surgery for upper and lower gastrointestinal malignancy in the meta-analyses reviewed. They analysed 27 RCTs published between 1996 and 2011 which included 2,538 patients. Seven RCTs compared the effects of preoperative enteral immunonutrition (EIN) diet versus SEN. The analysis showed that the IN intervention effectively decreased the incidence of postoperative infectious complications. Fifteen RCTs comparing the effects of postoperative EIN and SEN once again showed significant less infectious complications with the EIN compared to SEN. Six RCTs compared the effects of perioperative EIN vs. SEN on infectious complications and once again found EIN to be superior to SEN. Lastly 3 RCTs compared the effects of pre- $v s$. perioperative EIN and SEN on postoperative infectious complications and found a comparable effect. These authors further investigated the role of these two nutritional regimens on postoperative non-infectious complications. Five RCTs showed that postoperative EIN does not decrease the incidence of postoperative non-infectious complications versus SEN. Six RCTs comparing perioperative EIN vs. SEN showed better outcome with EIN. Lastly the authors analysed the impact of EIN and SEN on LOS. Preoperative EIN compared to SEN did not decrease the LOS based on 7 studies. Fifteen RCTs compared postoperative EIN vs. SEN and the analysis showed EIN has a positive impact on the length of hospital stay compared to SEN. Six RCTs compared perioperative EIN vs. SEN and the results once again showed EIN to be better compared to SEN. Based on the above results, the authors concluded that EIN is superior to SEN and the perioperative regimen is the optimum treatment option for patient undergoing gastrointestinal oncologic surgery.

Song et al. (25) produced a second meta-analysis looking at the role of EIN versus SEN on patients undergoing gastric cancer surgery. This was based on 9 RCTs published between 2002 and 2013 with a total of 785 patients. They found that although EIN was effective in enhancing host immunity, it did not have any impact on postoperative complications, hospital LOS, serum total protein and $\mathrm{CD}^{+}$. They pointed to the fact that heterogeneity caused by different compositions and timing of administration of EIN regimes and small sample size and number of eligible studies have an influence on the overall results of their analysis. The authors suggested that in the future only large scale well designed RCTs may resolve some of these lingering queries.

\section{Limitations}

There are considerable limitations inherent to all these meta-analyses investigating the effects of pharmaconutrition compared to standard composition nutrition support in elective gastrointestinal oncological surgical populations. Ultimately these are related to deficits in the reporting and/or methodologies of the included RCTs. First, few RCTs report on the amount of nutrition actually received relative to the documented nutritional goals in either the intervention or control groups: this forces readers to assume that nutrition goals were consistently met unless otherwise stated. This has obvious implications for the conclusions drawn, as reduced nutritional provision for reasons such as feed intolerance, non-compliance with oral supplements, tube-related complications or protocol deviations may have reduced the provision of nutrients and therefore may confound the results obtained. This aspect of reporting trials on pharmaconutrition needs to be addressed in future studies on this topic. Second, the majority of the pharmaconutrition studies-RCTs and systematic reviews/meta-analyses-have been funded at least in part by the companies that manufacture the products being investigated. This is of concern as funding bias is recognised for its potential to influence the results in favour of the product being investigated in pharmaceutical studies $(40,41)$. As meta-analysis is known to amplify biases included in the individual studies, the concern that funding bias may be present and has the potential to exaggerate the beneficial effects of pharmaconutrition should not be overlooked. This is of particular concern given the increasing acceptance that pharmaconutrition has found in clinical practice through its incorporation into clinical guidelines $(16,17)$. Interestingly, discussion of this aspect of pharmaconutrition is notably absent from the literature at the present time. Closely tied to concerns regarding funding bias is the frequent use of non-comparable control groups: this is a commonly observed trend in pharmaceutically funded studies that are subsequently shown to favour the intervention product (41). Significantly different protein contents between some of the intervention and control products were noted in several of the included studies. One such example is the Klek et al. (14) study that uses Peptisorb ${ }^{\circledR}$ (Nutricia Ltd., Poland) $(40 \mathrm{~g}$ protein $/ \mathrm{L} ; 1 \mathrm{kcal} / \mathrm{mL})$ as the control product against 
Stresson ${ }^{\circledR}$ (Nutricia Ltd., Poland) (75 g protein/L; 1.25 $\mathrm{kcal} / \mathrm{mL}$ ). While the lack of reporting of received nutrition make the significance of these differences on the current work impossible to evaluate, even in studies that utilize individualized nutritional goals based on caloric targets, such marked differences in formulations may ultimately undermine the controlled nature of individual studies due to the lack of appropriate control group. Fourth, reporting the prevalence and stratifying intervention groups for the presence and degree of malnutrition in the studied population is also imperative to differentiate the beneficial effects of pharmaconutrition interventions from the clinical impacts of treating of malnutrition. Complementary to this is the requirement for standardized or agreed definitions of malnutrition to be used in these studies to allow for valid comparison between studies regarding this issue. Finally, unavoidable heterogeneity is introduced by RCTs incorporated into meta-analyses that fail to separate out results of individual surgical procedures. This is significant as the complications likely to occur after procedures performed at various locations along the gastrointestinal tract vary greatly, and as such the indiscriminate grouping of these may confound the complications reported, and thus the effect attributed to the pharmaconutrition interventions provided.

\section{Conclusions}

So what can be concluded from these various meta-analyses, and which areas need to be highlighted and prioritized for future research on this topic? As described above, the impact of the nutritional reporting and selection of comparable control interventions is of great clinical importance and requires greater consideration in the design of future studies; without these essential areas being addressed, clinicians will not be able to make informed decisions regarding the most efficacious nutritional interventions in this patient group. Dietitians and nutrition professionals are largely absent from the authorship of the studies to date, and it seems likely that a more multidisciplinary approach to the research in this area is necessary, and is likely to alleviate these oversights in future studies. Secondly, convincing data supporting significant economic benefit related to the use of pharmaconutrition over standard nutrition products remains scarce in the literature. A strong body of evidence supporting the cost-benefit analysis of pharmaconutrition is going to be increasingly vital to justify its continued use in healthcare environments that are increasing subjected to financial scrutiny in these difficult economic times.
Drawing together the key points from this review of theses meta-analyses, the timing of pharmaconutrition provision is perhaps the most important clinical consideration regarding this issue at the present time. Two meta-analyses $(22,24)$ have shown that preoperative pharmaconutrition failed to deliver any benefit over standard formulations when used as a standalone intervention, and the accepted benefits of pharmaconutrition i.e., reduction in infectious complications and LOS, were only reported in peri- and postoperative administration. These two metaanalyses have further shown benefits of pharmaconutrition with respect to reduced non-infective complications and anastomotic dehiscence in postoperative and perioperative administration respectively. Based on the present evidence, it seems premature to include blanket recommendations for the use of pharmaconutrition within this population group without reference to the timing to provision.

\section{Future directions}

For future research it is essential that better quality, multidisciplinary, large scales well designed RCTs ought to be undertaken to further validate various beneficial effects of pharmaconutrition unequivocally without the funding of any pharmaceutical industry (manufacturers of various pharmaconutrition products) to separate the facts from the fictions. Furthermore, cost-effective studies need to be encouraged to further clarify (I) the direct and indirect cost savings for treating the gastrointestinal malignancy patients with pharmaconutrition; (II) the effectiveness of these intervention in treating these patients; (III) the cost savings with an equal or better health outcome following a major gastrointestinal oncological surgery and lastly (IV) having an additional benefit of pharmaconutrition worth the additional cost.

\section{Acknowledgements}

None.

\section{Footnote}

Conflicts of Interest: The authors have no conflicts of interest to declare.

\section{References}

1. Andersen HK, Lewis SJ, Thomas S. Early enteral 
nutrition within $24 \mathrm{~h}$ of colorectal surgery versus later commencement of feeding for postoperative complications. Cochrane Database Syst Rev 2006;(4):CD004080.

2. Osland E, Yunus RM, Khan S, et al. Early versus traditional postoperative feeding in patients undergoing resectional gastrointestinal surgery: a meta-analysis. JPEN J Parenter Enteral Nutr 2011;35:473-87.

3. Jones NE, Heyland DK. Pharmaconutrition: a new emerging paradigm. Curr Opin Gastroenterol 2008;24:215-22.

4. Daly JM, Weintraub FN, Shou J, et al. Enteral nutrition during multimodality therapy in upper gastrointestinal cancer patients. Ann Surg 1995;221:327-38.

5. Braga M, Gianotti L, Radaelli G, et al. Perioperative immunonutrition in patients undergoing cancer surgery: results of a randomized double-blind phase 3 trial. Arch Surg 1999;134:428-33.

6. Gianotti L, Braga M, Gentilini O, et al. Artificial nutrition after pancreaticoduodenectomy. Pancreas 2000;21:344-51.

7. Braga M, Gianotti L, Vignali A, et al. Preoperative oral arginine and n-3 fatty acid supplementation improves the immunometabolic host response and outcome after colorectal resection for cancer. Surgery 2002;132:805-14.

8. Braga M, Gianotti L, Nespoli L, et al. Nutritional approach in malnourished surgical patients: a prospective randomized study. Arch Surg 2002;137:174-80.

9. Farreras N, Artigas V, Cardona D, et al. Effect of early postoperative enteral immunonutrition on wound healing in patients undergoing surgery for gastric cancer. Clin Nutr 2005;24:55-65.

10. Xu J, Zhong Y, Jing D, et al. Preoperative enteral immunonutrition improves postoperative outcome in patients with gastrointestinal cancer. World J Surg 2006;30:1284-9.

11. Schilling J, Vranjes N, Fierz W, et al. Clinical outcome and immunology of postoperative arginine, omega-3 fatty acids, and nucleotide-enriched enteral feeding: a randomized prospective comparison with standard enteral and low calorie/low fat i.v. solutions. Nutrition 1996;12:423-9.

12. McCarter MD, Gentilini OD, Gomez ME, et al. Preoperative oral supplement with immunonutrients in cancer patients. JPEN J Parenter Enteral Nutr 1998;22:206-11.

13. Klek S, Kulig J, Sierzega M, et al. Standard and immunomodulating enteral nutrition in patients after extended gastrointestinal surgery--a prospective, randomized, controlled clinical trial. Clin Nutr
2008;27:504-12.

14. Klek S, Kulig J, Sierzega M, et al. The impact of immunostimulating nutrition on infectious complications after upper gastrointestinal surgery: a prospective, randomized, clinical trial. Ann Surg 2008;248:212-20.

15. Gunerhan Y, Koksal N, Sahin UY, et al. Effect of preoperative immunonutrition and other nutrition models on cellular immune parameters. World J Gastroenterol 2009;15:467-72.

16. Weimann A, Braga M, Harsanyi L, et al. ESPEN Guidelines on Enteral Nutrition: Surgery including organ transplantation. Clin Nutr 2006;25:224-44.

17. Carey S, He L, Ferrie S. Nutritional management of patients undergoing major upper gastrointestinal surgery: A survey of current practice in Australia. Nutr Diet 2010;67:219-23.

18. Waitzberg DL, Saito H, Plank LD, et al. Postsurgical infections are reduced with specialized nutrition support. World J Surg 2006;30:1592-604.

19. Marik PE, Zaloga GP. Immunonutrition in high-risk surgical patients: a systematic review and analysis of the literature. JPEN J Parenter Enteral Nutr 2010;34:378-86.

20. Cerantola Y, Hübner M, Grass F, et al. Immunonutrition in gastrointestinal surgery. Br J Surg 2011;98:37-48.

21. Zheng Y, Li F, Qi B, et al. Application of perioperative immunonutrition for gastrointestinal surgery: a metaanalysis of randomized controlled trials. Asia Pac J Clin Nutr 2007;16 Suppl 1:253-7.

22. Osland E, Hossain MB, Khan S, et al. Effect of timing of pharmaconutrition (immunonutrition) administration on outcomes of elective surgery for gastrointestinal malignancies: a systematic review and meta-analysis. JPEN J Parenter Enteral Nutr 2014;38:53-69.

23. Hegazi RA, Hustead DS, Evans DC. Preoperative standard oral nutrition supplements vs immunonutrition: results of a systematic review and meta-analysis. J Am Coll Surg 2014;219:1078-87.

24. Song GM, Tian X, Zhang L, et al. Immunonutrition support for patients undergoing surgery for gastrointestinal malignancy: preoperative, postoperative, or perioperative? A Bayesian network meta-analysis of randomized controlled trials. Medicine (Baltimore) 2015;94:e1225.

25. Song GM, Tian X, Liang H, et al. Role of Enteral Immunonutrition in Patients Undergoing Surgery for Gastric Cancer: A Systematic Review and Meta-Analysis of Randomized Controlled Trials. Medicine (Baltimore) 2015;94:e1311.

26. Heys SD, Walker LG, Smith I, et al. Enteral nutritional 
supplementation with key nutrients in patients with critical illness and cancer: a meta-analysis of randomized controlled clinical trials. Ann Surg 1999;229:467-77.

27. Heyland DK, Novak F, Drover JW, et al. Should immunonutrition become routine in critically ill patients? A systematic review of the evidence. JAMA 2001;286:944-53.

28. Beale RJ, Bryg DJ, Bihari DJ. Immunonutrition in the critically ill: a systematic review of clinical outcome. Crit Care Med 1999;27:2799-805.

29. Higgins JP, Green S, editors. Appendix 8a Considerations and recommendations for figures in Cochrane reviews: Graphs of statistical data. Cochrane Handbook for Systematic Reviews of Interventions 4.2.6 (updated September 2006); Appendices. Chichester, UK: John Wiley \& Sons Ltd., 2006.

30. Finco C, Magnanini P, Sarzo G, et al. Prospective randomized study on perioperative enteral immunonutrition in laparoscopic colorectal surgery. Surg Endosc 2007;21:1175-9.

31. Braga M, Gianotti L, Cestari A, et al. Gut function and immune and inflammatory responses in patients perioperatively fed with supplemented enteral formulas. Arch Surg 1996;131:1257-64; discussion 1264-5.

32. Daly JM, Lieberman MD, Goldfine J, et al. Enteral nutrition with supplemental arginine, RNA, and omega-3 fatty acids in patients after operation: immunologic, metabolic, and clinical outcome. Surgery 1992;112:56-67.

33. Wu GH, Zhang YW, Wu ZH. Modulation of

\section{doi: $10.21037 / \operatorname{tgh} .2018 .07 .12$}

Cite this article as: Osland E, Memon B, Memon MA. Pharmaconutrition administration on outcomes of elective oncological surgery for gastrointestinal malignancies: is timing everything? - a review of published meta-analyses until the end of 2016. Transl Gastroenterol Hepatol 2018;3:52. postoperative immune and inflammatory response by immune-enhancing enteral diet in gastrointestinal cancer patients. World J Gastroenterol 2001;7:357-62.

34. Klek S, Sierzega M, Szybinski P, et al. The immunomodulating enteral nutrition in malnourished surgical patients - a prospective, randomized, double-blind clinical trial. Clin Nutr 2011;30:282-8.

35. Jiang XH, Li N, Zhu WM, et al. Effects of postoperative immune-enhancing enteral nutrition on the immune system, inflammatory responses, and clinical outcome. Chin Med J (Engl) 2004;117:835-9.

36. Daly JM, Reynolds J, Thom A, et al. Immune and metabolic effects of arginine in the surgical patient. Ann Surg 1988;208:512-23.

37. Chen DW, Wei Fei Z, Zhang YC, et al. Role of enteral immunonutrition in patients with gastric carcinoma undergoing major surgery. Asian J Surg 2005;28:121-4.

38. Paccagnella A, Morassutti I, Rosti G. Nutritional intervention for improving treatment tolerance in cancer patients. Curr Opin Oncol 2011;23:322-30.

39. Kingham TP, Pachter HL. Colonic anastomotic leak: risk factors, diagnosis, and treatment. J Am Coll Surg 2009;208:269-78.

40. Chopra SS. MSJAMA: Industry funding of clinical trials: benefit or bias? JAMA 2003;290:113-4.

41. Lexchin J, Bero LA, Djulbegovic B, et al. Pharmaceutical industry sponsorship and research outcome and quality: systematic review. BMJ 2003;326:1167-70. 